\title{
Caspase inhibition supports proper gene expression in ex vivo mouse limb cultures
}

\author{
D De Valck ${ }^{1}$ and FP Luyten ${ }^{*, 1}$ \\ ${ }^{1}$ Laboratory of Skeletal Development and Joint Disorders, Department of \\ Rheumatology, University Hospitals, K.U. Leuven, Belgium \\ * Corresponding author: FP Luyten, Laboratory of Skeletal Development and \\ Joint Disorders, K.U. Leuven, Campus Gasthuisberg, Building “O\&N”, \\ Herestraat 49, B-3000 Leuven, Belgium. \\ Tel: (32) 16 346166; Fax: (32) 16 346200; \\ E-mail: frank.luyten@uz.kuleuven.ac.be
}

Received 30.11.00; revised 2.5.01; accepted 16.5.01

Edited by M Piacentini

\begin{abstract}
We standardized conditions for ex vivo mouse limb culture to study cartilage maturation and joint formation. We compared 12.5 d.p.c. mouse forelimbs that were cultured either mounted or freely rotating for up to $72 \mathrm{~h}$. Limb outgrowth progressed ex vivo at a variable rate as compared to its development in vivo, spanning approximately $48 \mathrm{~h}$. Although cartilage maturation and joint formation developed grossly normal, aberrant expression of skeletal marker genes was seen. Interestingly, no regression of the interdigital webs took place in mounted cultures, in contrast to limited webbing under freely rotating conditions. Caspase inhibition, by addition of ZVAD-fmk to the culture medium of freely rotating limbs, supported proper gene expression associated with skeletal development, and prevented interdigital web regression. Taken together, a freely rotating ex vivo culture for mouse limb outgrowth that is combined with caspase inhibition provides a good model to study cartilage maturation and joint formation. Cell Death and Differentiation (2001) 8, 985-994.
\end{abstract}

Keywords: mouse limb outgrowth; cartilage maturation; joint formation; interdigital web regression; caspase inhibition

Abbreviations: abc1, ATP binding cassette transporter-1; ALP, alkaline phosphatase; BMP, bone morphogenetic protein; c1qa, complement component 1qa; caspase, cysteine aspartic acid specific protease; col2a1, collagen type 2a1; d.p.c., days post coitum; $g d f 5$, growth and differentiation factor- 5 ; TGF- $\beta$, transforming growth factor- $\beta$; TUNEL, terminal deoxynucleotidyl transferasemediated dUTP-nick end labeling; zVAD-fmk, benzyl-oxycarbonylVal-Ala-Asp(O-methyl)-fluoromethylketone

\section{Introduction}

At midgestation of mouse embryonic development, limb outgrowth $^{1}$ provides an interesting model to study cartilage maturation, joint formation and webbing. Interdigital web regression, a prominent morphogenetic event, is the ultimate result of apoptosis of the interdigital tissues. For a long period, webbing has only been connected to the shaping of the limbs. ${ }^{2}$ However, recently it has been demonstrated that interdigital tissues also establish digit identification, ${ }^{3}$ prior to their regression. Simultaneously, mesenchymal cells condense inside the outgrowing limb to form the cartilaginous templates of the skeleton. ${ }^{4}$ Interestingly, while mesenchymal condensations lie down as full rays, the resulting matured cartilaginous elements are interrupted at specific sites by future joint interzones. ${ }^{5,6}$ The rapid expansion of molecular approaches in the field of developmental biology, including in situ hybridization, allowed identification of signaling pathways associated with these morphogenetic events. Morphogens involved in these processes include members of the Transforming Growth Factor- $\beta$ /Bone Morphogenetic Protein (TGF- $\beta / \mathrm{BMP}$ ) and Wnt superfamilies, as well as retinoic acid. $^{5-11}$

Data revealing the expression patterns of genes that are possibly involved in skeletal and joint development have to be expanded with functional studies, ultimately defining their biological relevance. Up to date, loss-of-function studies have been performed via gene targeting (knockouts, knock-ins). Conventional knock-outs are often embryonically lethal even before the onset of skeletal development, or can yield a phenotype that masks the latter process. Therefore it may be desirable to use conditional knock-outs with tissue specific promoter elements. However, gene targeting remains elaborative, expensive and time consuming, which hampers its routine use. Overexpression models (transgenes) address gain-offunction: we successfully generated transgene animals carrying the gene encoding cartilage-derived morphogenetic protein-1/growth and differentiation factor-5 (gdf5) controlled by the collagen type $2 a 1$ (col2a1) or col11a2 promoters, ${ }^{12}$ allowing overexpression of the gene specifically in cartilage. So far, tissue specific promoter elements that allow gene targeting/overexpression in diarthrodal joints are still lacking. One can anticipate that promoter elements $^{13}$ of joint specific markers such as gdf5 ${ }^{14}$ can drive expression of exogenous genes in the joint interzone.

In avian species, functional studies can be conveniently carried out using in ovo manipulation of the embryonic limb: for instance beads soaked in morphogens can be implanted, or the limb can be infected ${ }^{15}$ using retrovirus. Such a manipulated embryo can further be incubated and grown under physiological conditions until analysis of the skeletal phenotype. Despite the practical advantages associated with in ovo manipulation of avian embryos, it remains necessary to study mammalian limb outgrowth, since the molecular cascades involved in skeletal and joint development differ in part between these animal classes. $^{16-19}$ In the past decades, developmental biologists and teratologists already made sporadic attempts to apply ex vivo mouse limb outgrowth to perform functional 
studies. $^{20-24}$ However, this model has never been well standardized. Although the data obtained could be interpreted conclusively at the histological level, the validation of the model using molecular techniques such as in situ hybridization appears to be limited. ${ }^{24}$ Recently, ex utero surgical procedures for mouse limb manipulation have also been reported. ${ }^{25,26}$ However, it is a very tedious and technically demanding procedure not readily suitable for routine use to date.

Here we report on the standardization of the culture conditions for ex vivo mouse limb outgrowth. Our data reveal that cartilage maturation and joint formation can develop grossly normal in explanted limbs, independently of interdigital web regression. We furthermore demonstrate that caspase inhibition supports proper expression of skeletal marker genes, as analyzed by in situ hybridization. The described model allows future functional studies.

\section{Results}

\section{Standardization of culture conditions for ex vivo mouse limb outgrowth}

To develop standardized serum free culture conditions for ex vivo mouse limb outgrowth, we compared the two most commonly used setups. Therefore, 12.5 days post coitum (d.p.c.) mouse forelimbs were incubated either mounted or freely rotating (Figure 1). The limbs were allowed to further develop for up to $72 \mathrm{~h}$. Interestingly, inspection of the general morphology of limbs in mounted cultures indicated the absence of interdigital web regression (Figure 1, upper panel) in contrast to limited regression using freely rotating conditions (Figure 1, lower panel). As outlined in the forthcoming section, time kinetic analysis revealed that the observed interdigital web regression under freely rotating conditions occurred within $24 \mathrm{~h}$, but did not proceed thereafter (Figures 2, 3 and 5). Supply of additional nutrients such as $10 \%$ fetal bovine serum did not significantly improve the culture conditions. Also, preliminary experiments suggested that enhanced oxygen tension $\left(\begin{array}{lll}45 \% & \mathrm{O}_{2}\end{array}\right)^{27}$ does not significantly improve the outcome of ex vivo mouse limb culture, especially with respect to webbing and apoptosis of the ectoderm (data not shown).

\section{Different morphogenetic events progress ex vivo at a variable rate as compared to their development in vivo}

Inspection of the general morphology of explanted limbs already indicated that different morphogenetic events developed differentially during mounted or freely rotating culture conditions. Therefore, we compared cartilage maturation and joint formation in more detail to their development in vivo. We examined the maturation of mesenchymal condensations into cartilaginous elements separated by future joint interzones histologically by toluidine blue staining on cultured limbs versus limbs collected between 12.5 and 15.5 d.p.c. in vivo (Figure 2). In both culture conditions, cartilage maturation and establishment of future joint interzones progressed ex vivo to a developmental stage that is comparable to 14.5 d.p.c. in vivo,
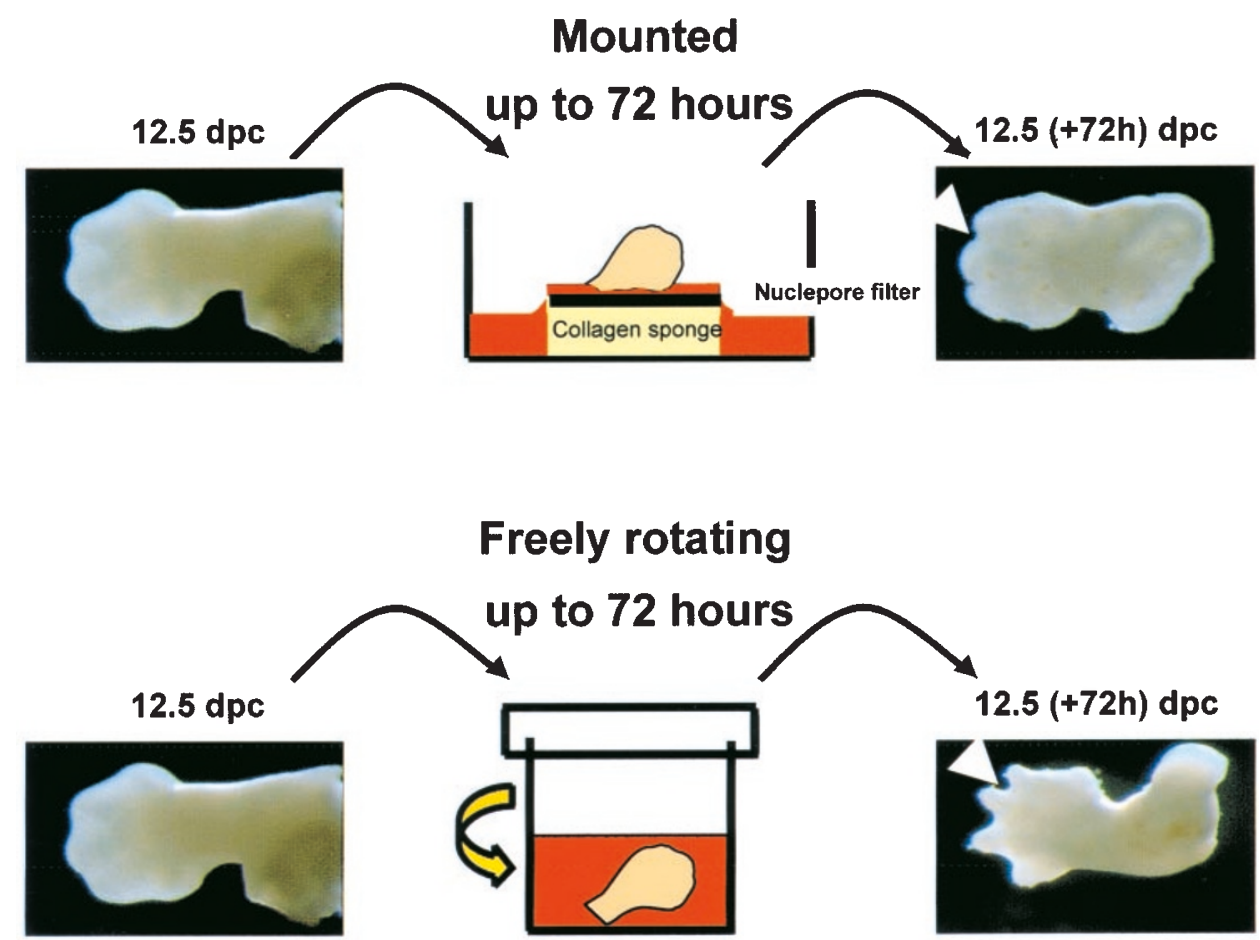

Figure 1 Schematic representation of the conditions used to develop a standardized model for ex vivo mouse limb culture. 12.5 d.p.c. mouse forelimbs were cultured either mounted (upper panel) or freely rotating (lower panel). General morphology of representative limbs is shown before (left) and after (right) $72 \mathrm{~h}$ of culture. Arrowheads point at interdigital tissues 
spanning approximately $48 \mathrm{~h}$. The mesenchymal condensations underwent differentiation into chondrocytes that produced an extracellular matrix composed of sulfated proteoglycans, and moreover gave rise to cartilaginous elements that retained grossly their normal shape.

The maturation of the cartilaginous elements is followed by endochondral ossification completing the developmental process that creates the calcified skeleton. ${ }^{28}$ Since the appearance of primary ossification centers within cartilaginous elements can be observed at 14.5 d.p.c. in vivo, we next examined if mouse limbs grown ex vivo can also undergo endochondral ossification. To detect the early onset of osteogenesis, we visualized histochemically alkaline phosphatase (ALP) activity ${ }^{29}$ (Figure 3). As expected, endogenous ALP activity could be detected in the perichondria and in the primary ossification centers of cartilaginous elements of limbs cultured up to $72 \mathrm{~h}$, irrespective of the conditions used. Even more pronounced than in the process of cartilage maturation, the upregulation of endogenous ALP activity followed a proximo-distal pattern. However, the pattern of ALP-positive cells in the perichondria of cultured limbs was often broadened and sometimes extended into the interdigital tissue, in contrast to the sharply delignated ALP-positive perichondria that border cartilaginous elements in vivo.

In addition to histological and histochemical analysis, we performed whole mount in situ hybridization on explanted limbs to detect the spatio-temporal expression of skeletal marker genes during culture (Figure 4). We chose to analyze col2a1 expression in chondroblasts and chondrocytes of the cartilaginous skeleton, ${ }^{30}$ as well as gdf5 expression in cells restricted to the narrowing joint interzones. ${ }^{14}$ First, the previously described expression patterns of these marker genes were reproduced in limbs collected between 12.5 and 15.5 d.p.c. in vivo. In contrast, aberrant gene expression patterns were observed in limbs cultured ex vivo. Although col2a1 expression could still be detected in the cartilaginous elements of the digits and radius/ulna, its expression was almost absent in the carpal/ metacarpal region. Furthermore, gdf5 was expressed barely above the detection limit and the pattern no longer reflected its expression in vivo.

\section{Caspase inhibition supports proper expression of skeletal marker genes}

Most likely, the failure to detect proper gene expression patterns in explanted limbs could be attributed to cell death of the tissue. Therefore, we performed a terminal deoxynucleotidyl transferase-mediated dUTP-nick end labeling (TUNEL) assay to detect nuclear DNA fragmentation in cells that undergo apoptosis ${ }^{31}$ (Figure 5). At 12.5 d.p.c. of mouse limb outgrowth in vivo, apoptotic cells could be detected in the interdigital tissues and more discrete within future joint interzones. When interdigital web regression proceeds between 13.5 and 15.5 d.p.c., a small number of apoptotic cells remained present underneath the ectoderm, as well as in the joint interzones. In limbs that were cultured ex vivo, the presence of TUNEL-positive cells was more pronounced and increased dramatically in time. The staining was no longer restricted to the interdigital tissues and future joint interzones, but was more abundant in a core of soft tissue located at the carpal/metacarpal region of the limb.

Despite the presence of apoptotic cells in the interdigital tissues of limbs that were cultured mounted, webbing did not proceed. This observation prompted us to investigate if clearance of apoptotic bodies was affected using this culture condition. Phagocytosis of cellular debris by
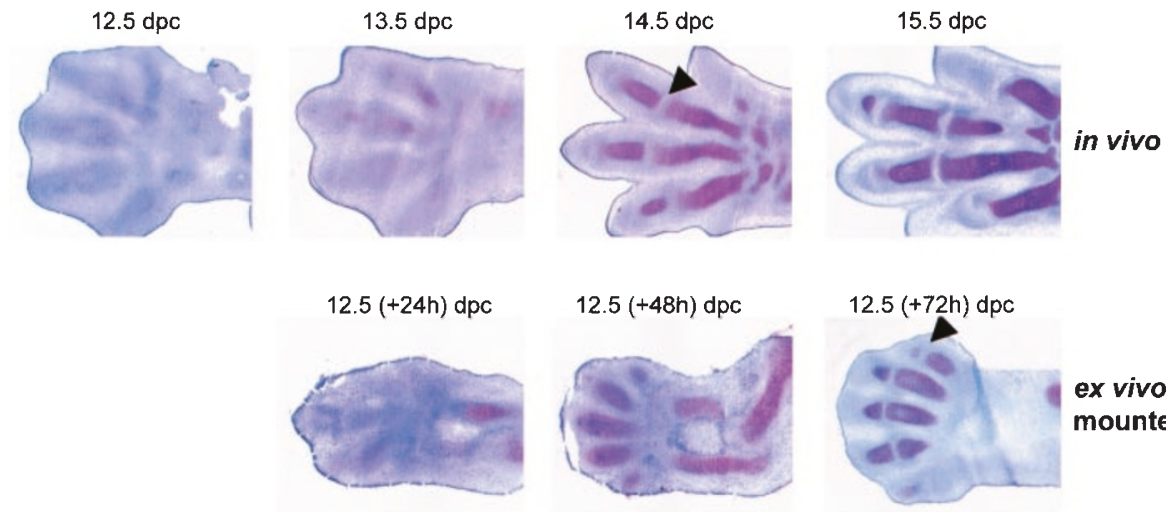

ex vivo

mounted
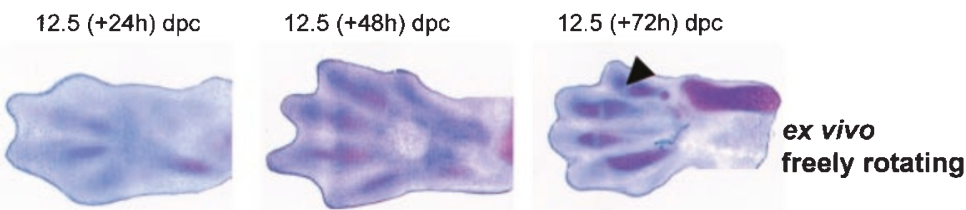

Figure 2 Cartilage maturation and formation of joint interzones during ex vivo mouse limb culture as compared to their development in vivo. Mouse forelimbs collected between 12.5 and 15.5 d.p.c. in vivo or 12.5 d.p.c. mouse forelimbs cultured for 24,48 and $72 \mathrm{~h}$ ex vivo using mounted or freely rotating conditions were cryosectioned and stained with toluidine blue. Arrowheads point at joint interzones 

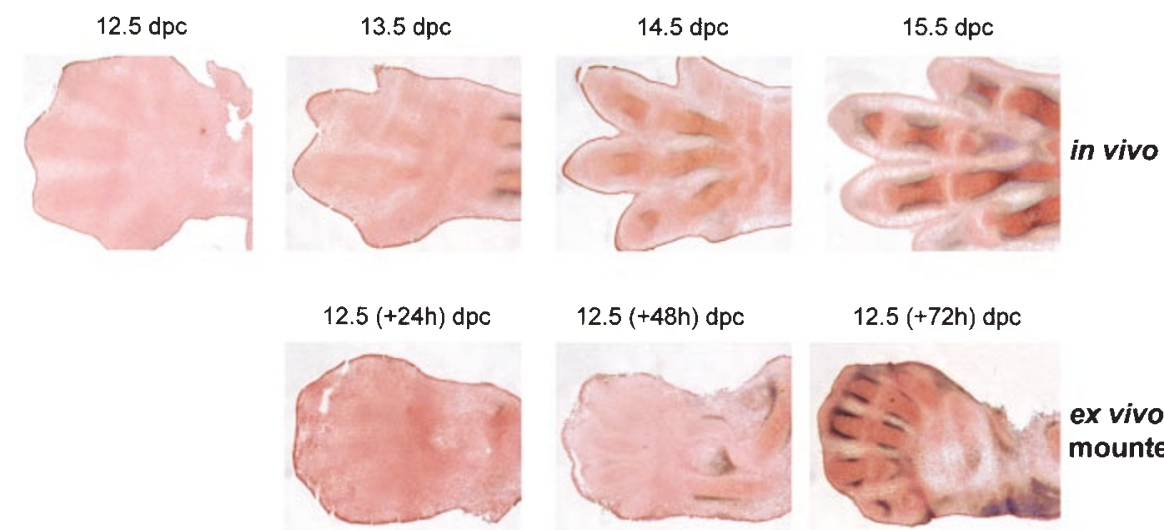

ex vivo

mounted
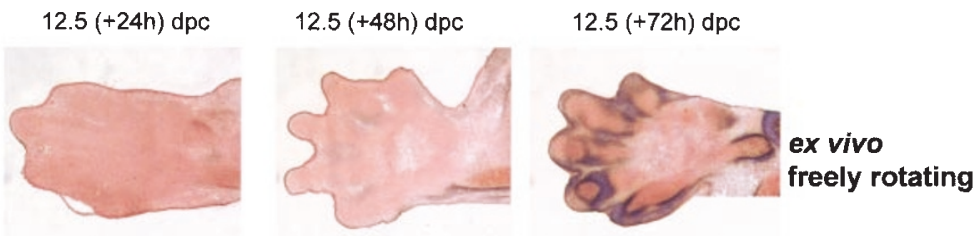

Figure 3 Onset of osteogenesis during ex vivo mouse limb culture as compared to its development in vivo. Histochemical ALP activity was performed on cryosectioned mouse forelimbs collected between 12.5 and 15.5 d.p.c. in vivo or on cryosectioned 12.5 d.p.c. mouse forelimbs cultured for 24,48 and $72 \mathrm{~h}$ ex vivo using mounted or freely rotating conditions

macrophages requires expression of various engulfing genes. Therefore, we analyzed by in situ hybridization the expression patterns of the molecular marker genes ATP binding cassette transporter-1 (abc1) ${ }^{32,33}$ and complement component 1qa (c1qa) ${ }^{33,34}$ in explanted limbs as compared to counterparts in vivo (Figure 6). In agreement with the previously described expression patterns, both $a b c 1$ and c1qa transcripts were visualized within regressing interdigital tissues of 13.5 d.p.c. mouse forelimbs. In limbs cultured mounted, both genes were also highly expressed in the soft tissue of interdigital webs, resembling the localization of apoptotic bodies as detected by TUNEL assay. These data demonstrate that phagocytosis of apoptotic debris is likely to proceed well during ex vivo mouse limb culture, and cannot account for the lack of interdigital web regression using mounted conditions.

To preserve the soft tissue of explanted limbs from apoptosis, we added the cysteine aspartic acid specific protease (caspase) ${ }^{35}$ inhibitor benzyloxycarbonyl-Val-AlaAsp(O-methyl)-fluoromethylketone (zVAD-fmk) ${ }^{36,37}$ to the culture medium. Lack of interdigital web regression was observed in limbs that were cultured freely rotating in the presence of $20 \mu \mathrm{M}$ zVAD-fmk (Figures 4 and 7), in contrast to counterparts to which $0.04 \%$ DMSO was added as a vehicle control (data not shown). In agreement, TUNELassay performed on zVAD-fmk treated limbs showed a significant decrease in the number of apoptotic cells in the carpal/metacarpal region (Figure 7). When whole mount in situ hybridization for col2a1 and gdf5 was performed under these conditions, their proper gene expression patterns were observed (Figure 4). Indeed, col2a1 could now be detected additionally within the carpal/metacarpal region. Furthermore, gdf5 expression was visualized in a pattern that is restricted to the future joint interzones. In addition, the expression pattern of gdf5 became sharper delignated within these joint interzones, resembling the developmental stage 14.5 d.p.c. in vivo. Similar data, although less pronounced, were obtained using mounted culture conditions in the presence of $20 \mu \mathrm{M}$ zVAD-fmk (data not shown).

The lack of interdigital web regression in zVAD-fmk treated limbs appeared to be no consequence of cytotoxicity of the inhibitor. Indeed, cartilage maturation, formation of future joint interzones and onset of osteogenesis progressed similarly in treated versus non treated counterparts (Figure 7).

\section{Discussion}

In the present study, we standardized culture conditions for ex vivo mouse limb outgrowth. The conditions require mounted or freely rotated incubation in serum free medium under normal oxygen tension. Supply of additional nutrients such as $10 \%$ fetal bovine serum did not significantly improve the culture conditions. Previously published experiments ${ }^{27}$ indicated that increase in oxygen tension supports proper maturation of tegumental structures. Our preliminary experiments suggest that enhanced oxygen tension does not significantly improve the outcome of ex vivo mouse limb culture, especially with respect to webbing and apoptosis of the ectoderm. At the histological and histochemical level, both culture conditions allowed mesenchymal cell condensations to mature equally well into cartilaginous elements interrupted by future joint interzones. The absence or presence of gentle fluid flow during mounted or freely rotating conditions, did not influence the morphogenetic events studied during the initial phase of skeletal and joint development. These results are in 
agreement with former data obtained in paralysed chick embryos, ${ }^{38-40}$ showing minor effects of limb immobilization on cartilage maturation and no interference with the formation of future joint interzones. During later stages of chick embryonic development only, absence of joint cavitation was observed using paralyzed conditions, which has been attributed to fusion of opposing cartilaginous elements.

In contrast, interdigital web regression is the most prominent morphogenetic event that is altered during the ex vivo culture conditions. A recent study described that interdigital webs are involved in the specification of digit identity, demonstrating an early patterning function for this tissue before its regression. ${ }^{3}$ Persistence of the interdigital webs, even throughout complete adulthood, has been described in the natural mouse mutant Hammertoe. ${ }^{41}$ Despite the interesting observation that this phenotype can be rescued by addition of retinoic-acid, ${ }^{42}$ candidate genes disrupted by the mutation have only recently been identified. ${ }^{43}$ Furthermore, severe delay of interdigital web regression is encountered as part of the phenotype associated with Apaf1 knock-out mice. ${ }^{44,45}$ Apaf-1, the mammalian homolog of $C$. elegans CED-4, has been characterized as an adaptor molecule involved in the activation of specific caspases. ${ }^{35}$ Although necrosismediated interdigital web regression has also been described under particular experimental conditions, ${ }^{46}$ it is generally accepted that interdigital web regression is caused by caspase-mediated apoptosis. ${ }^{2}$ Interestingly, small cell permeable tripeptides have been designed that are broad spectrum inhibitors of caspases, ${ }^{36,37}$ although non-specific effects on cathepsin inhibition have recently been reported. ${ }^{47}$ In agreement with previous reports, ${ }^{48-50}$ our observation that addition of such an inhibitor to the medium of explanted limbs blocks interdigital web regres-
$12.5 \mathrm{dpc}$
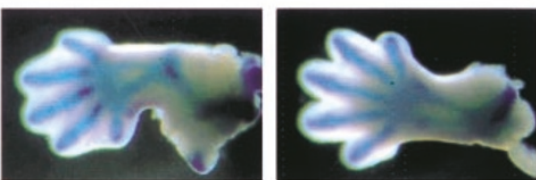

$12.5(+72 h) d p c$

col2a1

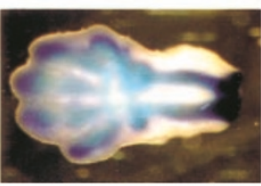

mounted

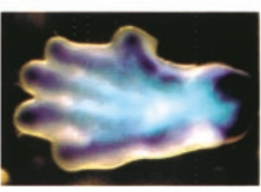

freely rotating
$14.5 \mathrm{dpc}$

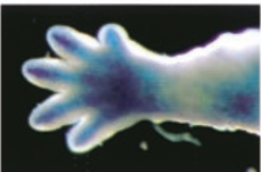

$12.5(+72 h) d p c$

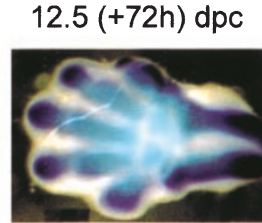

freely rotating

+ zVAD-fmk

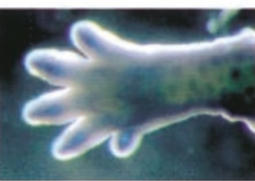

$12.5 \mathrm{dpc}$
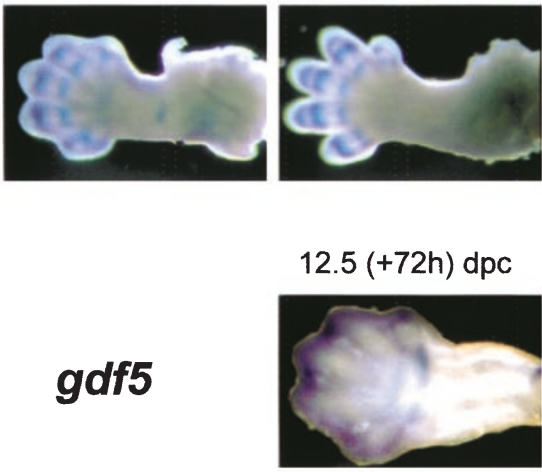

mounted

$13.5 \mathrm{dpc}$
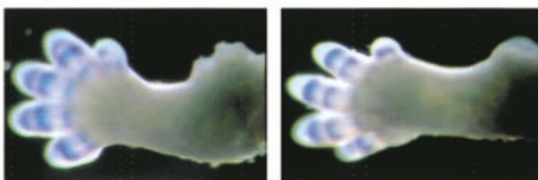

$12.5(+72 h) d p c$

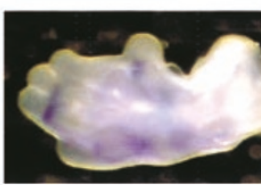

freely rotating

\section{ex vivo}

in vivo

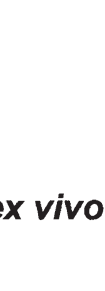

$15.5 \mathrm{dpc}$

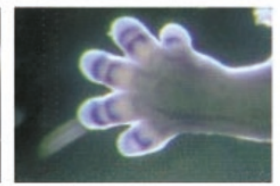

in vivo

$12.5(+72 h) d p c$

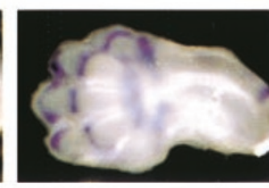

ex vivo

\section{freely rotating}

+ zVAD-fmk

Figure 4 Caspase inhibition supports proper expression of skeletal marker genes in ex vivo mouse limb cultures. Whole mount in situ hybridization with col2a1 (upper panel) or gdf5 (lower panel) probes was performed on mouse forelimbs collected between 12.5 and 15.5 d.p.c. in vivo or on 12.5 d.p.c. mouse forelimbs cultured for $72 \mathrm{~h}$ ex vivo using mounted conditions or freely rotating conditions in the absence or presence of $20 \mu \mathrm{M}$ zVAD-fmk 

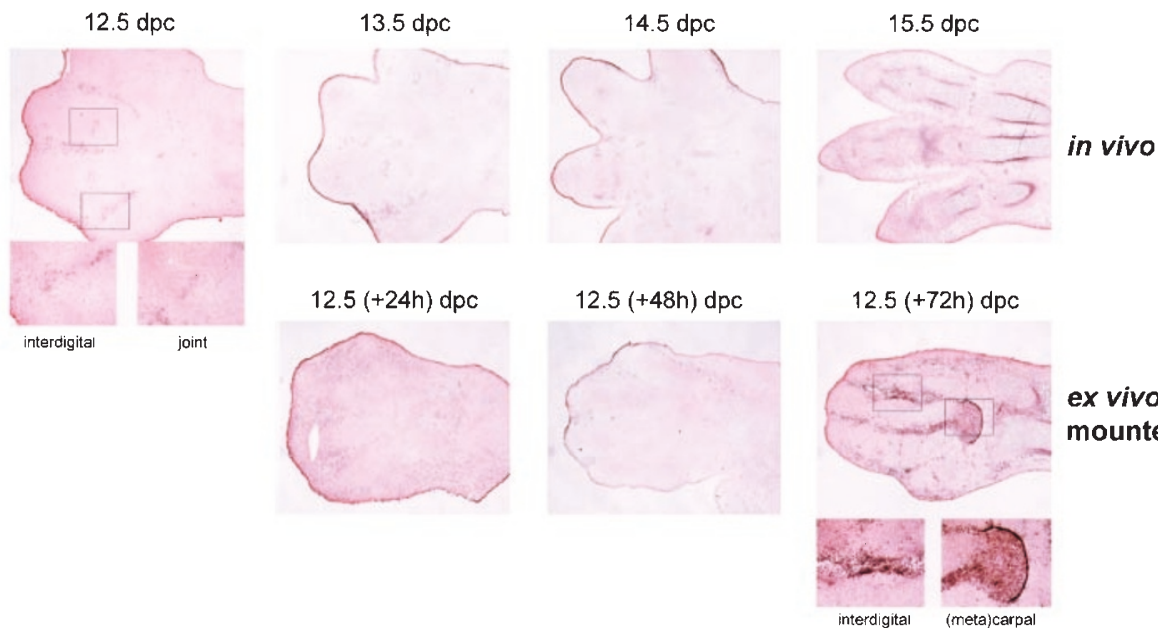

ex vivo mounted
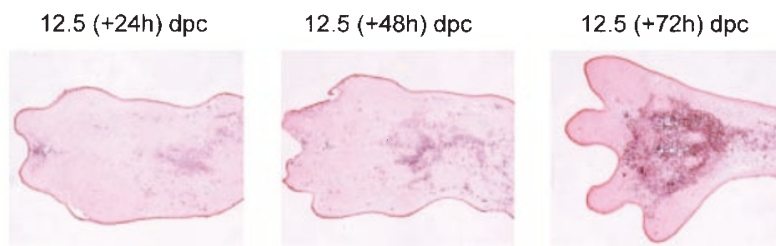

ex vivo

freely rotating

Figure 5 Presence of apoptosis during ex vivo mouse limb culture as compared to its detection during development in vivo. TUNEL assay was performed on cryosectioned mouse forelimbs collected between 12.5 and 15.5 d.p.c. in vivo or on cryosectioned 12.5 d.p.c. mouse forelimbs cultured for 24,48 and $72 \mathrm{~h}$ ex vivo using mounted conditions or freely rotating conditions

sion further points to the involvement of caspases in this process. Interestingly, digit individualization in developing limbs in culture can also be prevented by addition of antioxidants, suggesting that generation of reactive oxygen species participates also in the control of embryonic cell death. ${ }^{51}$

At present, the underlying mechanism by which interdigital web regression is completely or partially blocked during mounted or freely rotating culture conditions is unclear. In every respect, the pathway leading to apoptosis proceeds correctly, since even increased numbers of TUNEL-positive cells could be detected within the interdigital tissues of explanted limbs, as compared to counterparts in vivo. Also, phagocytosis that allows clearance of cellular debris upon apoptosis appeared not to be affected during ex vivo mouse limb culture. Macrophage recruitment to interdigital tissues during limb outgrowth in $v i v o^{52}$ is required for optimal engulfment of apoptotic bodies. ${ }^{53}$ Transbilayer redistribution of phosphatidylserine on the membranes of both phagocyte and prey is indispensible for the proper recognition of the cellular corpses, which is at least partly mediated by the activity of the $A B C 1$ transporter protein. Similar to our observations, an increased number of TUNEL-positive particles could be visualized within the interdigital tissues of $A B C 1$-deficient mouse limbs. ${ }^{54}$ Furthermore, macrophage-mediated phagocytosis of apoptotic bodies requires lysosomal activity, which may be affected by the culture conditions used. Indeed, a recent study revealed that immunohistochemical detection of the lysosomal membrane glycoprotein LAMP-1 in limbs that were subjected to ex vivo culture could be correlated to the degree of digit separation. ${ }^{55}$ Interestingly, it was recently described that phagocytosis of apoptotic debris in the developing limb can be taken over by 'standin' mesenchymal neighbors in the absence of functional macrophages. ${ }^{33}$

The main advantage of our 'upgraded' model for ex vivo mouse limb outgrowth above systems previously used by others, is the correct gene expression pattern analyzed by in situ hybridization. This technique is nowadays critical in the field of modern developmental biology and allows interpretation of data at the molecular level. Previous attempts to perform functional studies on skeletal and joint development in explanted mouse limbs have been hampered once solid conclusions had to be made beyond the histological level. ${ }^{24}$ Some investigators could circumvent these limitations by dissecting out the separate skeletal elements from the surrounding soft tissue, ${ }^{56}$ which excludes however possible biological interactions between different cell types in a physiological environment.

Taken together, the standardization of culture conditions for ex vivo mouse limb outgrowth revealed that skeletal and joint development can progress independently of interdigital web regression. The model is further validated by the observation that caspase inhibition supports proper gene expression. Therefore, the presented 'upgraded' model for ex vivo mouse limb outgrowth may now be applied as a fast and reliable assay to screen the biological relevance of novel molecules possibly involved in skeletal and joint development, prior to or in conjunction with functional studies using transgene and gene targeting technology. 


\section{Materials and Methods}

\section{Mouse embryonic limbs}

Embryos were collected between stages 12.5 and 15.5 d.p.c. from pregnant CD1 mice. Noon of the presence of a vaginal plug after natural mating was designated as 0.5 d.p.c. The forelimbs were carefully dissected at the level of the elbow joint under a dissection microscope using microscissors.

\section{Ex vivo mouse limb organ culture}

Mouse embryonic limbs (12.5 d.p.c.) were cultured for up to $72 \mathrm{~h}$ in a humidified atmosphere $\left(37^{\circ} \mathrm{C}, 5 \% \mathrm{CO}_{2}\right)$. Two different culture conditions were used (Figure 1): limbs were mounted in a 24-well chamber on top of a Nuclepore polycarbonate filter (Costar, Badhoevedorp, The Netherlands) placed on a Helistat collagen sponge (Colla Tec, Plainsboro, NJ, USA) or alternatively cultured freely rotating ( 5 rounds per min, $45^{\circ}$ angle) in a bijou bottle (Bibby Sterilin, Staffordshire, UK). Sponges were soaked, or limbs were directly submerged, in $500 \mu \mathrm{l}$ of $\mathrm{BGJ}_{\mathrm{b}}$ medium (Gibco BRL, Merelbeke, Belgium) supplemented with an antibiotic mixture (penicillin (100 units $/ \mathrm{ml})$, streptomycin $(100 \mu \mathrm{g} / \mathrm{ml})$, amphotericin B $(0.25 \mu \mathrm{g} / \mathrm{ml})$; Gibco BRL) and bovine serum albumin $(1 \mathrm{mg} / \mathrm{ml}$; Serva, Heidelberg, Germany). In comparative studies, submerged limbs were flushed with a gas mixture enriched in oxygen ${ }^{27}\left(45 \% \mathrm{O}_{2} / 50 \% \mathrm{~N}_{2} / 5 \%\right.$ $\mathrm{CO}_{2}$ ), or $10 \%$ fetal bovine serum (Bio Whittaker, Verviers, Belgium) was added to the culture medium. The tripeptide caspase inhibitor zVAD-fmk was purchased from Biomol (Plymouth Meeting, PA, USA),

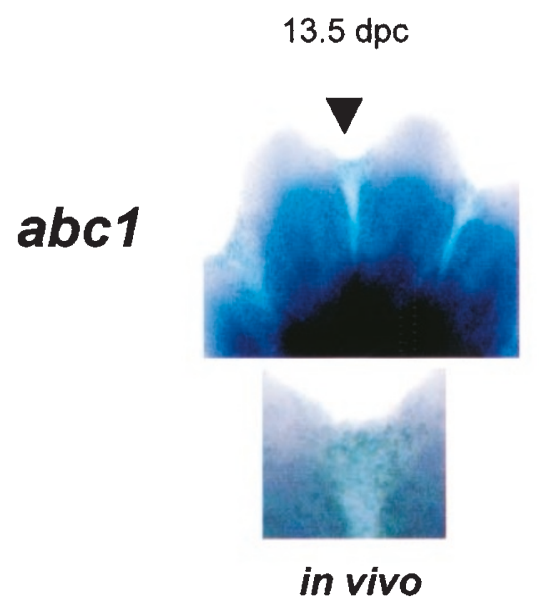

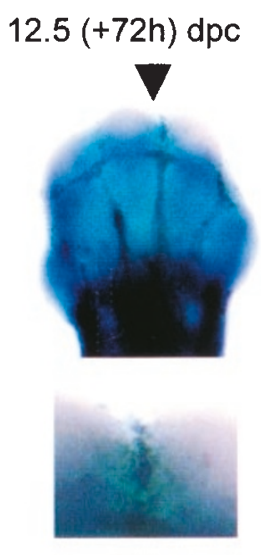

ex vivo

mounted
$12.5(+72 h) d p c$

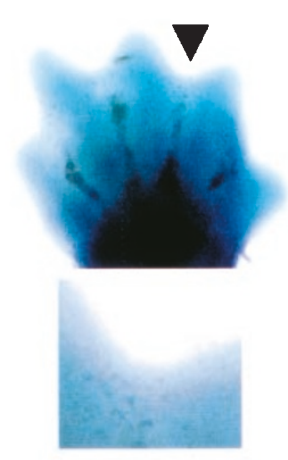

ex vivo

freely rotating
$13.5 \mathrm{dpc}$

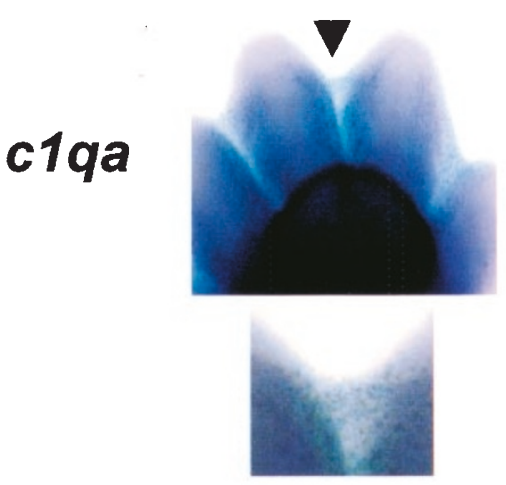

in vivo
$12.5(+72 h) d p c$
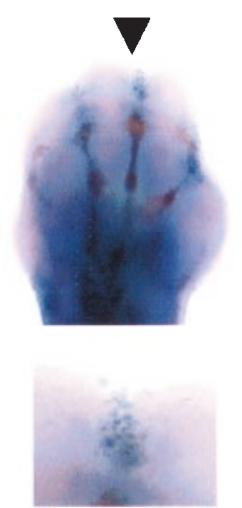

ex vivo

mounted
$12.5(+72 h) d p c$

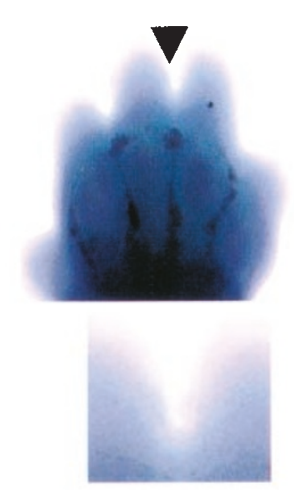

ex vivo

\section{freely rotating}

Figure 6 Presence of engulfing genes during ex vivo mouse limb culture as compared to its detection during development in vivo. Whole mount in situ hybridization with abc1 (upper panel) or c1qa (lower panel) probes was performed on mouse forelimbs collected at 13.5 d.p.c. in vivo or on 12.5 d.p.c. mouse forelimbs cultured for $72 \mathrm{~h}$ ex vivo using mounted or freely rotating conditions. Arrowheads point at positive staining in interdigital tissues 

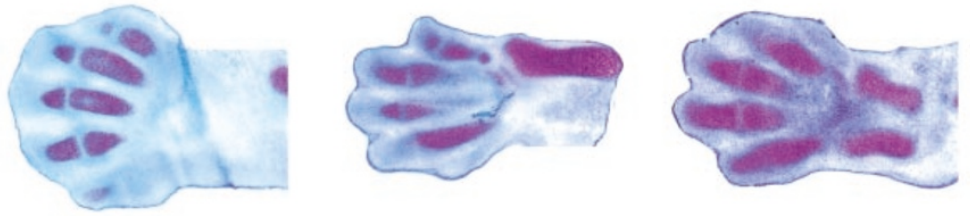

toluidine blue
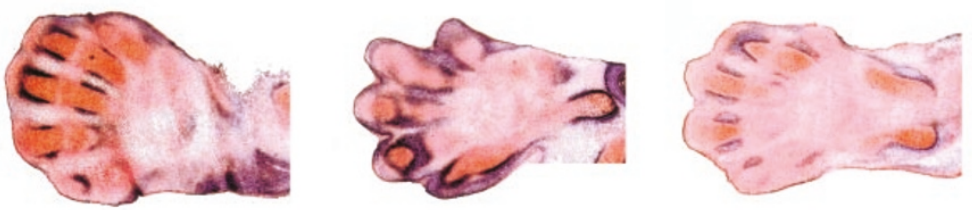

ALP activity
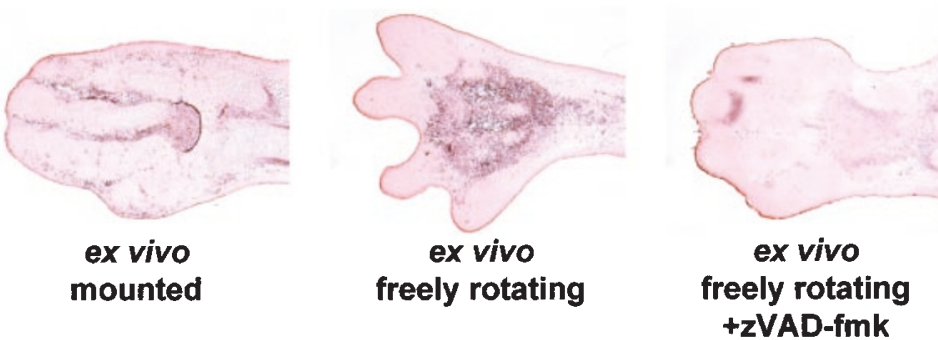

\section{TUNEL assay}

Figure 7 Caspase inhibition blocks interdigital web regression without affecting skeletal development in ex vivo mouse limb cultures. 12.5 d.p.c. mouse forelimbs were cultured for $72 \mathrm{~h}$ ex vivo using mounted or freely rotating conditions in the absence or presence of $20 \mu \mathrm{M} \mathrm{zVAD}$-fmk and cryosectioned. Cartilage maturation and formation of joint interzones was visualized by toluidine blue staining. Onset of osteogenesis was revealed by histochemical ALP activity. Apoptotis was detected using TUNEL assay

diluted in DMSO to a $50 \mathrm{mM}$ stock solution and applied as a $20 \mu \mathrm{M}$ final concentration.

\section{Histology, histochemical ALP activity and TUNEL assay}

Mouse embryonic limbs were processed for cryostat sectioning by snap freezing in Optimal Cutting Temperature compound (TissueTek $^{(\mathbb{R}}$; Sakura Finetek Europe, Zoeterwoude, The Netherlands) and stored at $-70^{\circ} \mathrm{C}$ until use. Frozen tissue sections were cut at $12 \mu \mathrm{m}$ and fixed in $4 \%$ paraformaldehyde. Metachromatic staining of sulfated proteoglycans in the extracellular cartilage matrix was performed by toluidine blue (Fluka, Bornem, Belgium). Endogenous ALP activity, a marker for the onset of osteogenesis, ${ }^{29}$ was detected using NBT/BCIP (Roche Molecular Biochemicals, Brussels, Belgium) as a substrate. Cells undergoing apoptosis were visualized using a TUNEL assay ${ }^{31}$ (ALP-based In Situ Cell Death detection kit; Roche Molecular Biochemicals) according to the manufacturer's instructions in the presence of $2 \mathrm{mM}$ levamisole (Sigma, Bornem, Belgium) to inhibit endogenous ALP activity. Except for toluidine blue stained sections, tissues were counterstained with neutral red (Klinipath, Turnhout, Belgium).

\section{Whole mount in situ hybridization}

Mouse embryonic limbs were fixed in MEMFA (0.1 M MOPS $\mathrm{pH} 7.4$, $2 \mathrm{mM}$ EGTA, $1 \mathrm{mM} \mathrm{MgSO}_{4}, 3.7 \%$ formaldehyde), transferred to $90 \%$ methanol and stored at $-20^{\circ} \mathrm{C}$ until use. Digoxigenin-11-UTP-labeled RNA probes were prepared using a DIG RNA labeling kit (Roche Molecular Biochemicals). Linearized plasmids containing cDNA of murine col2a1 ${ }^{30}$ (a 405 bp EcoRI-Hindlll fragment of the $3^{\prime} U T R$; gift from Dr. Suneel Apte, Cleveland Clinic Foundation, Cleveland, $\mathrm{OH}$, USA), murine gdf5 ${ }^{14}$ (a $1150 \mathrm{bp}$ Hindll-Apal fragment of the proregion), murine $a b c 1^{32}$ (a $1450 \mathrm{bp} X b a l-E c o R I$ fragment; gift from Dr. Giovanna Chimini, Inserm CNRS, Marseille, France) and murine $c 1 q a^{34}$ (a 540 bp Dral-BstYl fragment; gift from Dr. Marina Botto, Hammersmith Hospital, London, UK) were applied as templates in these reactions. Whole mount in situ hybridization was performed using a standard protocol. ${ }^{57}$ Probes were hybridized at $60^{\circ} \mathrm{C}$ and posthybridization washes were carried out to a final stringency of $0.2 \times \mathrm{SSC}$ at $65^{\circ} \mathrm{C}$. Visualization of gene expression was obtained using an ALP-coupled anti-DIG antibody and BM Purple (Roche Molecular Biochemicals) as a substrate in the presence of $2 \mathrm{mM}$ levamisole (Sigma). Prior to photography, residual background staining was cleared by rinsing the limbs in a series of $0.5 \% \mathrm{KOH}$ in glycerol $(0,25,50$ and $75 \%)$ for 15 min each.

\section{Acknowledgements}

The authors thank Dr. Suneel Apte (Cleveland Clinic Foundation, Cleveland, $\mathrm{OH}, \mathrm{USA}$ ) and Dr. Paul Martin (University College London, London, UK) for the generous gifts of col2a1, abc1 and c1qa cDNAs (courtesy of Dr. Giovanna Chimini and Dr. Marina Botto). Dr. An Zwijsen 
(Laboratory for Molecular Biology and Flanders Interuniversity Institute for Biotechnology) is acknowledged for laboratory animal facility service and assistance to the procedure of whole mount in situ hybridization. We thank Jenny Peeters and Giselle De Geest (Center for Human Genetics and Flanders Interuniversity Institute for Biotechnology) for technical advice on tissue sectioning. Dirk De Valck is a postdoctoral research assistant with the Fund for Scientific Research-Flanders. Research was supported by the Fund for Scientific Research-Flanders (grant $\mathrm{nr}$ G.0200.99) and the National Lottery (grant $\mathrm{nr}$ 9.0200.99).

\section{References}

1. Martin P (1990) Tissue patterning in the developing mouse limb. Int. J. Dev. Biol. 34: $323-336$

2. Chen $Y$ and Zhao X (1998) Shaping limbs by apoptosis. J. Exp. Zool. 282: 691 702

3. Dahn RD and Fallon JF (2000) Interdigital regulation of digit identity and homeotypic transformation by modulated BMP signaling. Science 289: $438-$ 441

4. Hall BK and Miyake T (2000) All for one and one for all: condensations and the initiation of skeletal development. BioEssays 22: 138-147

5. Dell'Accio F, De Bari C and Luyten FP (1999) Molecular basis of joint development. Jap. J. Rheumatol. 9: 17-29

6. Francis-West PH, Parish J, Lee K and Archer CW (1999) BMP/GDF-signalling interactions during synovial joint development. Cell. Tissue Res. 296: 111-119

7. Ng JK, Tamura K, Buscher D and Izpisua-Belmonte JC (1999) Molecular and cellular basis of pattern formation during vertebrate limb development. Curr. Top. Dev. Biol. 41: 37-66

8. Sandell LJ and AdlerP (1999) Developmental patterns of cartilage. Front. Biosci. 4: $731-742$

9. Monsoro-Burq AH and Le Douarin N (2000) Duality of molecular signaling involved in vertebral chondrogenesis. Curr. Top. Dev. Biol. 48: 43-75

10. Rodriguez-Leon J, Merino R, Macias D, Ganan Y, Santesteban E and Hurle JM (1999) Retinoic acid regulates programmed cell death through BMP signalling. Nat. Cell Biol. 1: 125-126

11. Dupé V, Ghyselinck NB, Thomazy V, Nagy L, Davies PJA, Chambon P and Mark $M$ (1999) Essential roles of retinoic acid signaling in interdigital apoptosis and control of BMP-7 expression in mouse autopods. Dev. Biol. 208: 30-43

12. Tsumaki N, Tanaka K, Arikawa-Hirasawa E, Nakase T, Kimura T, Thomas JT, Ochi T, Luyten FP and Yamada Y (1999) Role of CDMP-1 in skeletal morphogenesis: promotion of mesenchymal cell recruitment and chondrocyte differentiation. J. Cell. Biol. 144: 161-173

13. Sugiura T, Hotten $G$ and Kawai S (1999) Minimal promoter components of the growth/differentiation factor-5 gene. Biochem. Biophys. Res. Commun. 263: $707-713$

14. Storm EE and Kingsley DM (1996) Joint patterning defects caused by single and double mutations in members of the bone morphogenetic protein (BMP) family. Development 122: 3969-3979

15. Logan $C$ and Francis-West $P$ (1999) Gene transfer in avian embryos using replication-competent retroviruses. In Methods in molecular biology, Sharpe PT and Mason I, eds (Totowa, NJ: Humana Press) 539-551

16. Kengaku M, Capdevila J, Rodriguez-Esteban C, De La Pena J, Johnson RL, Izpisua-Belmonte JC and Tabin CJ (1998) Distinct Wnt pathways regulating AER formation and dorsoventral polarity in the chick limb bud. Science 280: $1274-1277$

17. Lescher B, Haenig B and Kispert A (1998) sFRP-2 is a target of the Wnt-4 signaling pathway in the developing metanephric kidney. Dev. Dyn. 213: 440451

18. Leimeister C, Bach A and Gessler M (1998) Developmental expression patterns of mouse sFRP genes encoding members of the secreted frizzled related protein family. Mech. Dev. 75: 29-42

19. Ladher RK, Church VL, Allen S, Robson L, Abdelfattah A, Brown NA, Hattersley G, Rosen V, Luyten FP, Dale L and Francis-West PH (2000) Cloning and expression of the Wnt antagonists Sfrp-2 and Frzb during chick development. Dev. Biol. 218: 183-198

20. Milaire $\mathrm{J}$ and Mulnard $\mathrm{J}$ (1984) Histogenesis in 11-day mouse embryo limb buds explanted in organ culture. J. Exp. Zool. 232: 359-377
21. Canoun C, Ma C, Halpern D, Shum L, Bringas P, Sank A and Slavkin HC (1993) Endogenous epidermal growth factor regulates limb development. J. Surg. Res. 54: $638-647$

22. Ginsburg GT, Royster D, Kassabian G, Shuler CF, Dougherty WR and Sank AC (1995) Mesenchymal commitment to digital joint formation. Ann. Plast. Surg. 35: 95-104

23. Stafford DL, Lussier MR, Sank AC and Shuler CF (1995) In vitro model of syndactyly replicates the morphological features observed in vivo. Plast. Reconstr. Surg. 96: 1169-1176

24. Storm EE and Kingsley DM (1999) GDF5 coordinates bone and joint formation during digit development. Dev. Biol. 209: 11-27

25. Tang MK, Leung AK, Kwong WH, Chow PH, Chan JY, Ngo-Muller V, Li Mand Lee KK (2000) Bmp-4 requires the presence of the digits to initiate programmed cell death in limb interdigital tissues. Dev. Biol. 218: 89-98

26. Ngo-Muller V and Muneoka K (2000) Influence of FGF4 on digit morphogenesis during limb development in the mouse. Dev. Biol. 219: 224-236

27. Zeltinger $\mathrm{J}$ and Holbrook KA (1997) A model system for long-term serum-free suspension organ culture of human fetal tissues: experiments on digits and skin from multiple body regions. Cell Tissue Res. 290: 51-60

28. Stevens DA and Williams GR (1999) Hormone regulation of chondrocyte differentiation and endochondral bone formation. Mol. Cell. Endocrinol. 151: $195-204$

29. Väkevä L, Mackie E, Kantomaa T and Thesleff I (1990) Comparison of the distribution patterns of tenascin and alkaline phosphatase in developing teeth, cartilage, and bone of rats and mice. Anta. Rec. 228: 69-76

30. Cheah KS, Lau ET, Au PK and Tam PP (1991) Expression of the alpha1(II) collagen gene is not restricted to cartilage during development. Development 111: $945-953$

31. Gold R, Schmied M, Giegerich G, Breitschopf H, Hartung HP, Toyka KV and Lassmann H (1994) Differentiation between cellular apoptosis and necrosis by the combined use of in situ tailing and nick translation techniques. Lab. Invest. 71: $219-225$

32. Luciani M-F and Chimini G (1996) The ATP binding cassette transporter ABC1 is required for the engulfment of corpses generated by apoptotic cell death. EMBO J. 15: $226-235$

33. Wood W, Turmaine M, Weber R, Camp V, Maki RA, McKercher SR and Martin P (2000) Mesenchymal cells engulf and clear apoptotic footplate cells in macrophageless PU.1 null mouse embryos. Development 127: 5245-5252

34. Botto M, Dell'Agnola C, Bygrave AE, Thompson EM, Cook HT, Petry F, Loos M, Pandolfi PP and Walport MJ (1998) Homozygous C1q deficiency causes glomerulonephritis associated with multiple apoptotic bodies. Nat. Genet. 19:56-59

35. Earnshaw WC, Martins LM and Kaufmann SH (1999) Mammalian caspases: structure, activation, substrates, and functions during apoptosis. Annu. Rev. Biochem. 68: 383-424

36. Zhu H, Fearnhead HO and Cohen GM (1995) An ICE-like protease is a common mediator of apoptosis induced by diverse stimuli in human monocytic THP.1 cells. FEBS Lett. 374: 303-308

37. Fearnhead HO, Dinsdale D and Cohen GM (1995) An interleukin-1 $\beta$-converting enzyme-like protease is a common mediator of apoptosis in thymocytes. FEBS Lett. 375: $283-288$

38. Mitrovic $D$ (1982) Development of the articular cavity in paralyzed chick embryos and in chick embryo limb buds cultured on chorioallantoic membranes. Acta Anat. (Basel) 113: 313-324

39. Persson M (1983) The role of movements in the development of sutural and diarthrodial joints tested by long-term paralysis of chick embryos. J. Anat. 137: $591-599$

40. Hosseini A and Hogg DA (1991) The effects of paralysis on skeletal development in the chick embryo. I. General effects. J. Anat. 177: 159-168

41. ZakeriZ, Guaglino D and Ahuja HS (1994) Apoptotic cell death in the mouse limb and its suppression in the hammertoe mutant. Dev. Biol. 165: 294-297

42. Ahuja HS, James $W$ and Zakeri Z (1997) Rescue of the limb deformity in hammertoe mutant mice by retinoic acid-induced cell death. Dev. Dyn. 208: 466-481

43. Clark RM, Marker PC and Kingsley DM (2000) A novel candidate gene for mouse and human preaxial polydactyly with altered expression in limbs of hemimelic extra-toes mutant mice. Genomics 67: 19-27

44. Cecconi F, Alvarez-Bolado G, Meyer BI, Roth KA and Gruss P (1998) Apaf1 (CED-4 homolog) regulates programmed cell death in mammalian development. Cell 94: 727-737 
45. Yoshida H, Kong Y-Y, Yoshida R, Elia AJ, Hakem A, Hakem R, Penninger JMand Mak TW (1998) Apaf1 is required for mitochondrial pathways of apoptosis and brain development. Cell 94: 739-750

46. Chautan M, Chazal G, Cecconi F, Gruss P and Golstein P (1999) Interdigital cell death can occur through a necrotic and caspase-independent pathway. Curr. Biol. 9: 967-970

47. Schotte P, Declercq W, Van Huffel S, Vandenabeele P and Beyaert R (1999) Non-specific effects of methyl ketone peptide inhibitors of caspases. FEBS Lett. 442: $117-121$

48. Milligan CE, Prevette D, Yaginuma H, Homma S, Cardwell C, Fritz LC, Tomasell KJ, Oppenheim RW and Schwartz LM (1995) Peptide inhibitors of the ICE protease family arrest programmed cell death of motoneurons in vivo and in vitro. Neuron 15: 385-393

49. Jacobson MD, Weil M and Raff MC (1996) Role of Ced-3/ICE-family proteases in staurosporine-induced programmed cell death. J. Cell. Biol. 133: 1041-1051

50. Lee KK, Tang MK, Yew DT, Chow PH, Yee SP, Schneider C and Brancolini C (1999) Gas2 is a multifunctional gene involved in the regulation of apoptosis and chondrogenesis in the developing mouse limb. Dev. Biol. 207: 14-25

51. Salas-Vidal E, Lomeli H, Castro-Obregon S, Cuervo R, Escalante-Alcalde and Covarrubias $L$ (1998) Reactive oxygen species participate in the control of mouse embryonic cell death. Exp. Cell Res. 238: 136-147
52. Hopkinson-Woolley J, Hughes D, Gordon S and Martin P (1994) Macrophage recruitment during limb development and wound healing in the embryonic and foetal mouse. J. Cell Sci. 107: 1159-1167

53. Savill J and Fadok V (2000) Corpse clearance defines the meaning of cell death. Nature 407: 784-788

54. Hamon Y, Broccardo C, Chambenoit O, Luciani M-F, Toti F, Chaslin S, Freyssinet J-M, Devaux PF, McNeish J, Marguet D and Chimini G (2000) ABC1 promotes engulfment of apoptotic cells and transbilayer redistribution of phosphatidylserine. Nat. Cell Biol. 2: 399-406

55. Stewart S, Yi S, Kassabian G, Mayo M, Sank A and Shuler C (2000) Changes in expression of the lysosomal membrane glycoprotein, LAMP-1 in interdigital regions during embryonic mouse limb development, in vivo and in vitro. Anat. Embryol. 201: 483-490

56. Haaijman A, D'Souza RN, Bronckers ALJJ, Goei SW and BurgerEH (1997) OP-1 (BMP-7) affects mRNA expression of type I, II, $\mathrm{X}$ collagen, and matrix gla protein in ossifying long bones in vitro. J. Bone Miner. Res. 12: 1815-1823

57. Hargrave $M$ and Koopman $P$ (2000) In situ hybridization of whole-mount embryos. In Methods in molecular biology, Darby IA, ed (Totowa, NJ: Humana Press) 279-289 